\title{
Entropy of level-cut random Gaussian structures at different volume fractions
}

\author{
Stjepan Marčelja \\ Department of Applied Mathematics, Research School of Physics \& Engineering, \\ Australian National University, Canberra, ACT 2601, Australia
}

(Received 6 August 2017; published 23 October 2017)

\begin{abstract}
Cutting random Gaussian fields at a given level can create a variety of morphologically different two- or several-phase structures that have often been used to describe physical systems. The entropy of such structures depends on the covariance function of the generating Gaussian random field, which in turn depends on its spectral density. But the entropy of level-cut structures also depends on the volume fractions of different phases, which is determined by the selection of the cutting level. This dependence has been neglected in earlier work. We evaluate the entropy of several lattice models to show that, even in the cases of strongly coupled systems, the dependence of the entropy of level-cut structures on molar fractions of the constituents scales with the simple ideal noninteracting system formula. In the last section, we discuss the application of the results to binary or ternary fluids and microemulsions.
\end{abstract}

DOI: 10.1103/PhysRevE.96.042147

\section{INTRODUCTION}

The random structures encountered in diverse fields ranging from microemulsions or porous rocks to astrophysics are often described with random Gaussian fields. The description can provide a variety of morphological structures, as the spectral density of the Gaussian field can be selected to match the particular application. When each spatial position can only be occupied by one of a two (or a few phases) the configuration can be described by one (or several) level cut(s) of the field, separating the different phases. Prominent early applications were in the description of spinodal decomposition [1,2] or microemulsion structures [3]. An example of the structures obtained as a level cut of a random Gaussian field shown in Fig. 1 was selected to resemble the surfactantfree microemulsions [4], where the spectral density has the Lorentzian form.

In earlier work [5-10], the dependence of the system entropy on the volume fraction of the components was neglected. The entropy of the structures obtained after the level cut was approximated by the entropy of the generating Gaussian field. The justification for the approximation was the fact that the generating field can be recovered from the information available from the level-cut state. This topic was discussed within the information theory [11], where it was found that the full reconstruction is possible under specific conditions.

However, as the fraction of the minor phase becomes smaller, such reconstruction requires ever-higher mathematical precision. The rationale for the approximation fails due to the discreteness of the physical space.

The entropy change with the fractional density can be studied by taking advantage of the correspondence of the two-state level-cut structures with the Ising models [12]. In the approximate Ising analogy, the Gaussian field covariance matrix with the elements $r_{i j}$ is related to the interaction strengths $V\left(r_{i j}\right)$ and the cutting level $\alpha$ corresponds to the external magnetic field $H$.

The analogy can be exploited to obtain insight from exactly solvable models. However, it is also useful to consider the mean-field approximation. Functional form of the dependence of the entropy on the applied magnetic field $H$ requires solving the familiar transcendental equation for the magnetization per site $m$ in order to obtain the mean magnetic field $H_{m f}$ :

$$
m=\tanh \left(\beta H_{m f}\right)=\tanh \left[\beta \Sigma V\left(r_{i j}\right) m+H\right] .
$$

At the same time, expressing the entropy per site in terms of magnetization is very simple as it involves only the definition of entropy in terms of the up- or down-spin probabilities. The mean-field entropy per site in units where $k_{B}=1$ is

$$
S=-\frac{1+m}{2} \ln \left(\frac{1+m}{2}\right)-\frac{1-m}{2} \ln \left(\frac{1-m}{2}\right) .
$$

In a lattice model of binary fluids, the magnetization is analogous to the difference between molar fractions $x_{1}$ and $x_{2}$ of the components, $m=x_{1}-x_{2}$. For the ideal mixture

$$
S=-x_{1} \ln x_{1}-\left(1-x_{1}\right) \ln \left(1-x_{1}\right),
$$

which is also the entropy of the ideal monoatomic lattice gas. The maximal value of the ideal entropy is $S=\ln 2$ or one bit per site, which is obtained when $x_{1}=x_{2}=\frac{1}{2}$. Correlations in the fluid will decrease the entropy, sometimes by orders of magnitude.

The relation between the cutting level $\alpha$ and the molar fraction of one constituent [3]

$$
x_{1}(\alpha)=\frac{1}{2} \operatorname{erfc} \frac{\alpha}{\sqrt{2}}
$$

may be used to obtain a more complicated expression for entropy in terms of $\alpha$.

In the following sections, we use several methods to show that even in the highly correlated lattice models obtained from level cuts of random Gaussian fields the entropy scales with the molar fraction of constituent phases according to the simple mean-field law, Eq. (3). In the last section, we relate the lattice model results to real binary or ternary fluids where constituent molecules are unequal in size and discuss the origin of the simple scaling of entropy with molar fractions. 
(a)

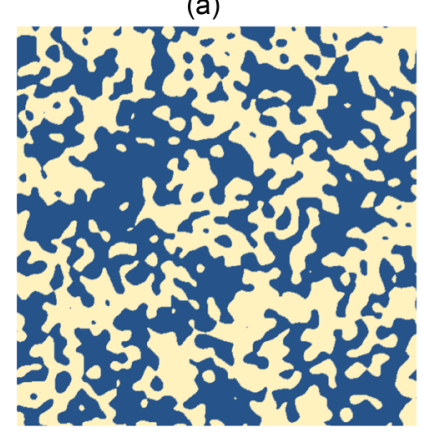

(b)

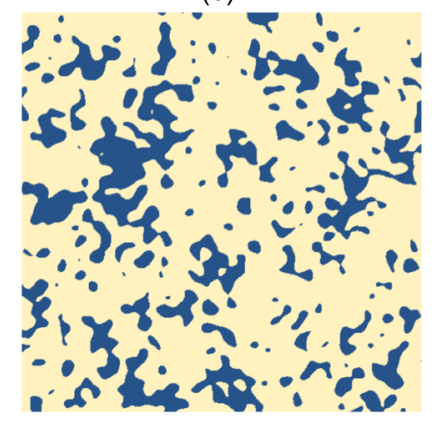

FIG. 1. A structure obtained by a level cut of a random Gaussian field with a Lorentzian spectrum. In (a) the fraction of each phase is $1 / 2$; in (b) the fractions are $1 / 3$ and $2 / 3$. We wish to find a change in the configurational entropy on going from (a) to (b).

\section{METHODS}

\section{A. Monte Carlo simulation}

In an earlier work [12] we used Monte Carlo (MC) simulation in order to study the entropy of one-dimensional lattice models of disordered systems with long-range interaction. The accuracy of simulations was established by testing against the exactly solvable Ising model with long-range interaction [13]. Applying the same simulation method to level-cut Gaussian fields it is possible to evaluate the entropy as a function of the strength and the range of correlations. For the purpose of testing, we selected the Lorentzian spectral distribution with the exponential covariance function

$$
K(r)=\exp (-\gamma|r|)
$$

This choice is demanding because of the relatively slow decay of correlations. Its spectral distribution,

$$
f(k)=\frac{\gamma}{\pi\left(\gamma^{2}+k^{2}\right)},
$$

corresponds to many physical structures. We used the same covariance function in all testing.

Entropy was evaluated as a function of the inverse correlation length $\gamma$ (Fig. 2). Also shown for comparison is the approximate evaluation of the entropy with small clusters (see below). In these one-dimensional examples, and using the segments of 18 or 19 sites, simulations easily reach four-figure accuracy. Due to the slow decay of Lorentzian correlations, simulations in two or three dimensions are difficult and were used only to verify the two-dimensional result from small cluster calculations.

\section{B. Exact entropy of small clusters}

The values of Gaussian random fields in space follow multivariate normal distribution, with the covariance matrix determined by the spectral density function. By integrating over the probability distribution we can find the probabilities for each of the possible states of a small cluster and evaluate the entropy. Applying the method developed by Aleksandrowicz and Meirovitch [14,15] one evaluates the entropy per site as the difference between the clusters of $n$ and $n-1$ sites. As an example, for a four-site cluster, $2^{4}$ possible states can be

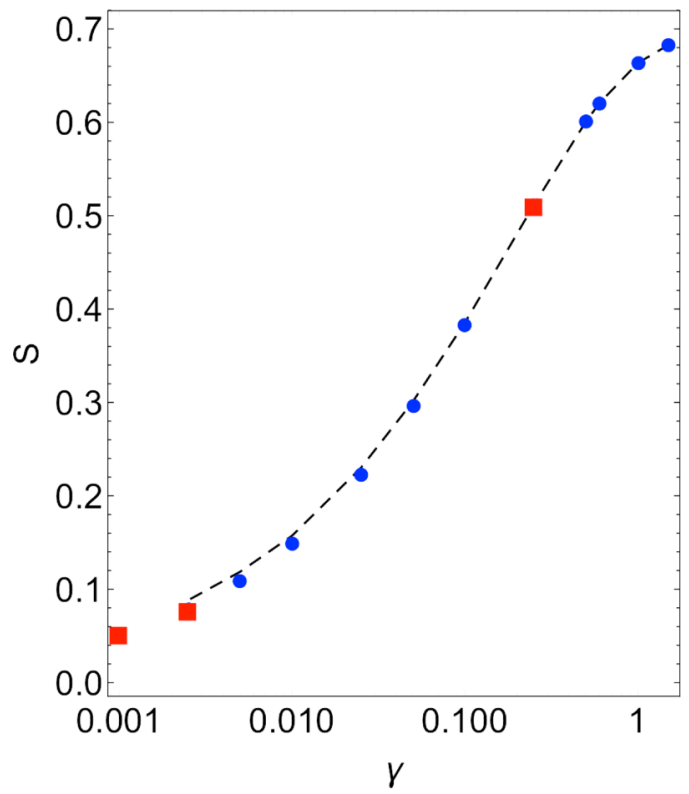

FIG. 2. Entropy per site for one-dimensional level-cut states evaluated by MC simulation (symbols) and by the small cluster approximation (dashed line). Relative entropy for the three marked points $(\gamma=0.25,0.0025$, and 0.001$)$ as a function of the fractional density is shown in Fig. 3.

enumerated as, e.g., 1001, where the successive digits mean that the value of the field at the corresponding site is above over below the cutting level $\alpha$. The associated probability for this state is

$$
\begin{aligned}
p_{1001}= & \frac{1}{(2 \pi)^{2} \sqrt{|\Sigma|}} \int_{\alpha}^{\infty} \int_{-\infty}^{\alpha} \int_{-\infty}^{\alpha} \int_{\alpha}^{\infty} \\
& \times \exp \left(-\frac{1}{2} x^{\mathrm{T}} \Sigma^{-1} \boldsymbol{x}\right) d^{4} x
\end{aligned}
$$

where $\boldsymbol{\Sigma}$ is the covariance matrix for the cluster configuration. The first integration can be performed analytically, but beyond that numerical methods are needed. The probabilities for some states are identical, reducing the number of integrations to fewer than 16. Many approximation methods have been proposed in literature. We found that the numerical integration within MATHEMATICA provides sufficient accuracy.

The entropy of the cluster is simply the sum over all the states

$$
S_{4}=-\sum_{1}^{16} p_{i j k l} \ln p_{i j k l},
$$

and the entropy per site is approximated as $S=S_{4}-S_{3}$.

Clusters with four sites can be formed in one, two or three dimensions, and this will be reflected in the form of the covariance matrix. For example, using the notation $x=\exp (-\gamma)$, the covariance matrix for an elementary threedimensional four-site cluster is

$$
\Sigma=\left(\begin{array}{cccc}
1 & x & x & x \\
x & 1 & x^{\sqrt{2}} & x^{\sqrt{2}} \\
x & x^{\sqrt{2}} & 1 & x^{\sqrt{2}} \\
x & x^{\sqrt{2}} & x^{\sqrt{2}} & 1
\end{array}\right) .
$$


The cluster approximation is particularly accurate for onedimensional arrays. The accuracy of simple approximations in one dimension was already observed in an earlier work where the entropy was approximated by the correlation function expansions [16]. In higher dimensions and for long-range correlations, entropy per site evaluated from small clusters will be higher than the entropy for infinite lattice. As shown in the next section, the relative entropy scaling rule remains very accurate.

\section{Binary digit count of compressed configuration files}

Algorithmic randomness, defined as the length of the shortest file containing the full description of a physical state, is equivalent to the Gibbs-Shannon entropy of this system [17]. Best commercial file compression engines achieve similar compression ratios. Testing with relatively disordered structures we found that most correlations in the compressed files are removed and the reduced bit count was close to the theoretical limit.

We created images of level-cut random Gaussian fields at a specified fractional density of the phases, similar to the images shown in Fig. 1. Files were then compressed (using the Stuffit engine by Smith Micro), and the length of the file header was subtracted from the result. The number of bits in the compressed files was close to the values obtained by other methods. The binary digit counting thus provides an alternative method to determine the dependence of entropy on the fractional density. In the next section (Fig. 5) it is shown that the simple law Eq. (3) is closely followed.

More generally, the binary count for common images reduced to the black and white format at a different fraction of black or white area also approximately follows the same scaling. As expected, the approximation is better for images with fewer high-order correlations.

\section{RESULTS}

\section{A. Binary systems}

The MC entropy of one-dimensional systems at three coupling strengths marked in Fig. 2 is shown in Fig. 3. Regardless of the interaction strength, and for very different absolute entropies, the relative entropy as a function of density perfectly follows the ideal law, Eq. (3). Figure 3(b) shows testing with clusters in two dimensions. The cluster method performs well at moderate correlation. At high correlation, it fails when one molar fraction is very small.

Absolute values of the entropy are smaller in three dimensions, as the correlation between sites in a cluster is stronger. The small cluster evaluation becomes inaccurate for higher correlation and very asymmetrical compositions. Nevertheless, as shown in Fig. 4, when the cluster approximation is accurate the relative entropy closely follows the ideal system behavior.

Last, we used the binary digit count of files containing twodimensional images of level-cut Gaussian fields at different area fractions (Fig. 5). The agreement for relatively disordered structures with weak high-order correlations as shown in Fig. 1 is almost perfect. The method can be applied to any image with a varying degree of accuracy. Even so, the simple law of Eq. (3) (a)

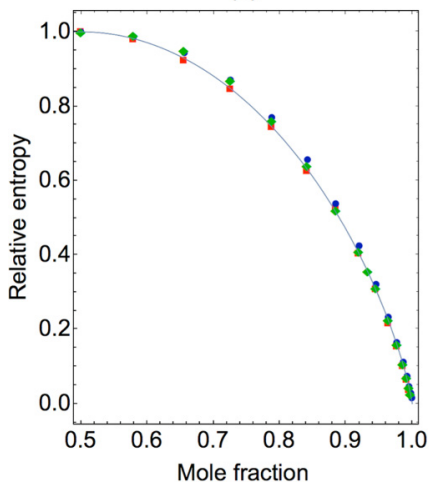

(b)

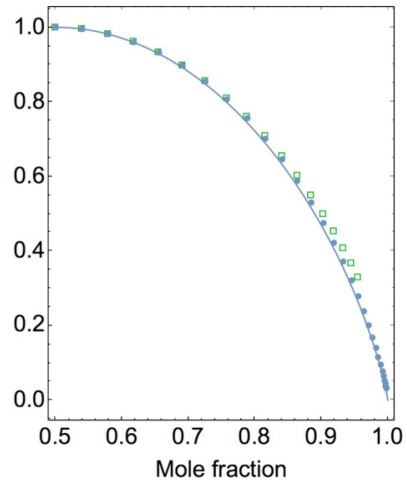

FIG. 3. (a) Relative entropy of one-dimensional systems evaluated in MC simulations at very different interaction strengths: $\gamma=0.25$ (circles), 0.0025 (diamonds), and 0.001 (squares). (b) Two-dimensional relative entropy evaluated as a difference between small cluster entropies for the interaction strength $\gamma=0.25$ (closed symbols) and $\gamma=0.05$ (open symbols). The $\gamma=0.25$ results were checked with MC simulations, which agreed with the data in the figure. In both parts, the full line follows Eq. (3).

is a useful first approximation for the information content of images reduced to two or several tones.

\section{B. Ternary systems}

The scaling of the relative entropy with molar fractions described above is not limited to binary systems. In particular, the generalization to ternary phases is straightforward. Such level-cut structures can be used to describe ternary solutions or microemulsions with three components. Examples of possible structures are shown in Fig. 6.

The entropy of an uncorrelated ternary system where each molecule occupies one lattice site or an ideal three-component monoatomic lattice gas in terms of molar fractions $x_{1}$ and $x_{2}$ is

$$
\begin{aligned}
S\left(x_{1}, x_{2}\right)= & -x_{1} \ln x_{1}-x_{2} \ln x_{2} \\
& -\left(1-x_{1}-x_{2}\right) \ln \left(1-x_{1}-x_{2}\right) .
\end{aligned}
$$

(a)

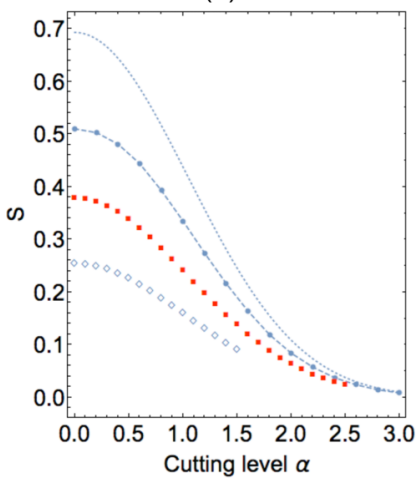

(b)

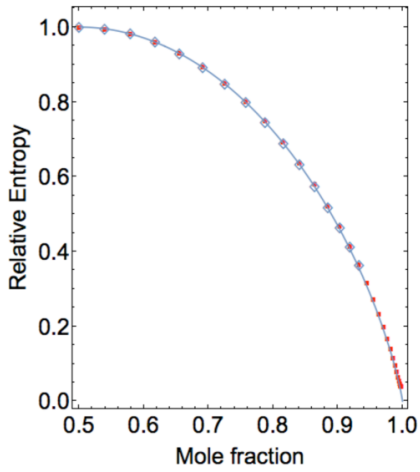

FIG. 4. (a) Entropy per site as a function of molar fraction as evaluated from three-dimensional clusters at interaction strengths $\gamma=0.25$ (circles), 0.1 (squares), and 0.05 (open diamonds). The top line is the entropy of noninteracting systems. (b) The four separate curves in (a) fall together when replotted as a relative entropy. 


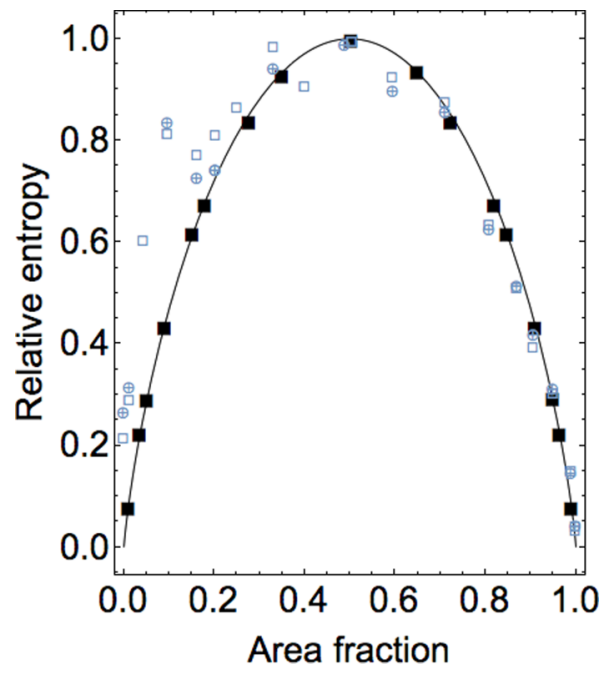

FIG. 5. Relative entropy estimated from the length of the compressed files. Filled squares are data points obtained from images of a level-cut random Gaussian field. Two such images are shown in Fig. 1. Other symbols are obtained from a standard test image "Lena" reduced to two tones. As the image contains high-order correlations, the agreement is very approximate. The compression engine works linearly and the results for two perpendicular orientations (different open symbols) are therefore similar but not identical.

The entropy of correlated ternary solutions approximately scales with molar fractions according to Eq. (10). Figure 7 illustrates this behavior along a particular line in a phase diagram where the ratio of components $\mathrm{B}$ and $\mathrm{C}$ is kept constant.

\section{DISCUSSION}

The application of level-cut random Gaussian fields to the description of different physical structures does not depend on lattice modeling. When the molecular volumes of constituents are equal, molar fractions and volume fractions are equal, each lattice site corresponds to a molecule of the solution, and the evaluated thermodynamic quantities are independent of the lattice.

For molecules of unequal size, the choice of a single lattice is an approximation subject to well-known restrictions [18]. Larger molecules may occupy more than one site, while in the case of polymers each segment is normally assigned to a lattice site. The simple and at the same time very successful result for the ideal mixing entropy in such systems is obtained by the Flory-Huggins theory [18]. For $\mathrm{n}_{1}$ and $\mathrm{n}_{2}$ molecules of components 1 and 2 respectively, the entropy of mixing is

$$
S=-k_{B}\left(n_{1} \ln v_{1}+n_{2} \ln v_{2}\right),
$$

where $v_{1}$ and $v_{2}$ are the respective volume fractions of the components in the mixture. Component 2 is typically a larger flexible molecule. Molar fractions in the arguments of the $1 n$ functions in the ideal law Eq. (3) are presently replaced by volume fractions. When the molecular volumes are equal, the approximate Eq. (11) reduces to Eq. (3). Equation (11) is still independent of any lattice parameters. The derivation is easily generalized to ternary systems [18], where Eq. (11) has the same form with three terms [19].

The structure of condensed matter solutions is normally constrained by numerous interactions, and the ideal law Eq. (11) then gives the upper limit for the entropy. The requirements are particularly prominent when amphiphilic or hydrotrope components are included, as this may lead to the formation of well-defined structures or aggregates. Gaussian random fields are useful here because the spectral density and the level cut can be adjusted to match the observed structures. The entropy is evaluated by integration in $q$-space involving the spectral density. The molecular size enters through the upper limit of the integration. When possible, the limit is best determined from the scattering data, e.g., as the high- $q$ limit of the observed Ornstein-Zernike behavior. The entropy is then estimated as the entropy of the field before the cut (as (a)

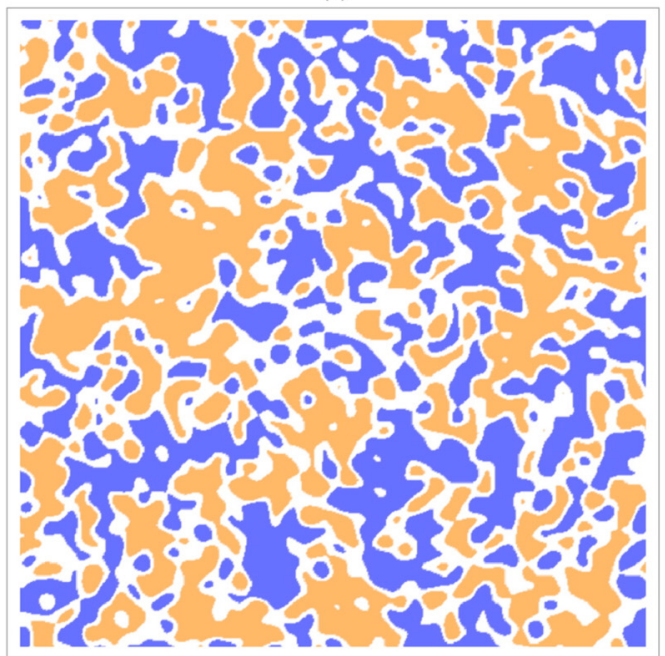

(b)

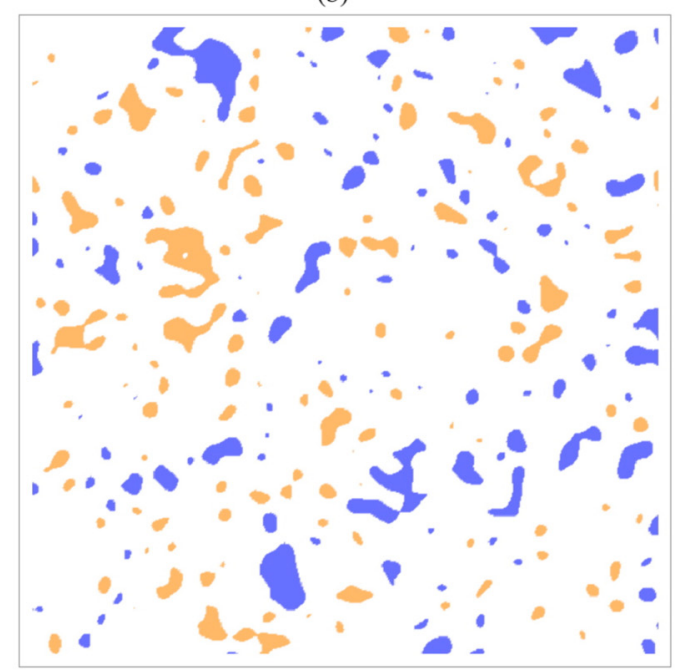

FIG. 6. Random Gaussian field cut at two levels. In (a), each of the three fractions occupies $1 / 3$ of the volume. In (b), each of the minor fractions occupies 0.063 of the volume. The generating random field is the same in both (a) and (b). 

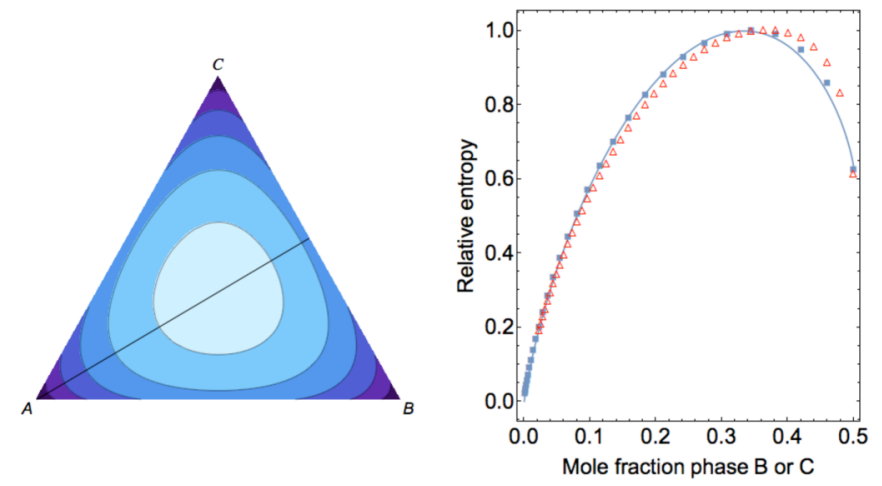

FIG. 7. Left: Contour plot of an ideal ternary system entropy, Eq. (10). Right: The relative entropy evaluated along the line originating in A. Evaluation is based on small clusters for $\gamma=$ 0.25 (closed symbols) and $\gamma=0.1$ (open symbols). The ideal law, Eq. (10), is shown as a full line.

in Refs. [4-10]) or evaluated from simulations. The results of this study suggest that the ideal law dependence on volume fractions applies approximately even in the case of correlated structures, and the estimates should scale with the volume fractions according to Eq. (11).

The approximate scaling of relative entropy of strongly coupled systems derived by level cuts of Gaussian fields is a remarkable property. Unlike the generating Gaussian fields, level-cut states do have correlations beyond the second order and would not be expected to follow the ideal gas behavior.

In order to make a plausible argument let us consider for the moment the correspondence between random Gaussian fields with a given spectral density and the binary level-cut states. If there are $\mathrm{N}$ molecules in the system of which $\mathrm{N} 1$ belong to the first constituent, the number of possible level-cut states is $\mathrm{N} ! /[\mathrm{N} 1 !(\mathrm{N}-\mathrm{N} 1) !]$. This number of states is the largest when molar fractions are equal and is reduced when $\mathrm{N} 1$ is increased or decreased from the value of $N / 2$. This is equally true for ideal and for correlated systems.

In the ideal case, all states are equally likely and the Boltzmann formula $S=k_{B} \ln W$ leads to Eq. (3). For correlated fluids, there are large differences in the probabilities. Nevertheless, the reduction in the number of possible states resulting from the departure from equal fractions is the same for both the ideal and the correlated systems. The level-cutting maps continuous Gaussian random fields onto a space with a fixed number of possible states. In this mapping, the entropy of correlated structures approximately scales with the number of available states following the law that describes the entropy of simple noninteracting systems.

\section{ACKNOWLEDGMENTS}

I am grateful to Thomas Zemb who led me to reconsider this topic and contributed valuable suggestions. Research was performed in part at the Institut de Chimie Séparative de Marcoule and funded by the European Research Council under the EU Seventh Framework Programme grant Rare Earth Recycling with Low Harmful Emissions (REE-CYCLE 320915).
[1] J. W. Cahn, Phase separation by spinodal decomposition in isotropic systems, J. Chem. Phys. 42, 93 (1965).

[2] N. F. Berk, Scattering properties of a model bicontinuous structure with a well-defined length scale, Phys. Rev. Lett. 58, 2718 (1987).

[3] M. Teubner, Level surfaces of Gaussian random fields and microemulsions, Europhys. Lett. 14, 403 (1991).

[4] T. N. Zemb, M. Klossek, T. Lopian, J. Marcus, S. Schöettl, D. Horinek, S. F. Prevost, D. Touraud, O. Diat, S. Marčelja, and W. Kunz, How to explain microemulsions formed by solvent mixtures without conventional surfactants, Proc. Natl. Acad. Sci. USA 113, 4260 (2016).

[5] P. Pieruschka and S. Marčelja, Statistical mechanics of random bicontinuous phases, J. Phys. II (France) 2, 235 (1992).

[6] P. Pieruschka and S. A. Safran, Random interfaces and the physics of microemulsions, Europhys. Lett. 22, 625 (1993).

[7] P. Pieruschka and S. A. Safran, Random interface model of sponge phases, Europhys. Lett. 31, 207 (1995).

[8] L. Arleth, S. Marčelja, and T. Zemb, Gaussian random fields with two level-cuts-Model for asymmetric microemulsions with nonzero spontaneous curvature?J. Chem. Phys. 115, 3923 (2001).

[9] M. Duvail, J.-F. Dufrêche, L. Arleth, and T. Zemb, Mesoscopic modelling of frustration in microemulsions, Phys. Chem. Chem. Phys. 15, 7133 (2013).

[10] M. Duvail, L. Arleth, T. Zemb, and J.-F. Dufrêche, Predicting for thermodynamic instabilities in water/oil/surfactant microemul- sions: A mesoscopic modelling approach, J. Chem. Phys. 140, 164711 (2014).

[11] S. R. Curtis and A. V. Oppenheim, Reconstruction of multidimensional signals from zero crossings, J. Opt. Soc. Am. 4, 221 (1987).

[12] S. Marčelja, Entropy of phase-separated structures, Physica A 231, 168 (1996).

[13] G. A. Baker, Jr., One-dimensional order-disorder model which approaches a second-order phase transition, Phys. Rev. 122, 1477 (1961).

[14] H. Meirovitch and Z. Alexandrowicz, The stochastic models method applied to the critical behavior of Ising lattices, J. Stat. Phys. 16, 121 (1997).

[15] H. Meirovitch, Methods for estimating entropy with computer simulation: The simple cubic Ising lattice, J. Phys. A 16, 839 (1983).

[16] P. Attard, O. G. Jepps, and S. Marčelja, Information content of signals using correlation function expansions of the entropy, Phys. Rev. E 56, 4052 (1997).

[17] W. H. Zurek, Algorithmic randomness and physical entropy, Phys. Rev. A 40, 4731 (1989).

[18] P. J. Flory, Principles of Polymer Chemistry (Cornell University Press, Ithaca, NY, 1953), Chap. XII.

[19] See Supplemental Material at http://link.aps.org/supplemental/ 10.1103/PhysRevE.96.042147 for an example of the ideal entropy of a ternary solution. 